\section{Embolismo gaseoso arterial cerebral y coronario}

\section{Arterial cerebral and coronary gas embolism}

Sr. Editor: El embolismo gaseoso vascular consiste en la entrada de gas en el interior de las vías arteriales o venosas y se deriva principalmente de procedimiento iatrogénicos, pudiendo llegar a producir importantes consecuencias médicas e incluso la muerte.

Presentamos el caso de una_paciente de 66 años de edad con antecedentes de hernia de hiato. Fue intervenida por un vólvulo gástrico y la cirugía se complicó con absceso supramesocólico y mediastinitis. Un mes después reingresó por dolor epigástrico y vómitos, en el tránsito gastroduodenal se objetivó una gran hernia hiatal paraesofágica con vólvulo gástrico completo. Se redujo el contenido del saco mediastínico que contenía el estómago, epiplón y colon transverso. Se realizó funduplicatura tipo Toupet, gastropexia a pared abdominal anterior e hialoplastia con malla bilaminar. El postoperatorio cursó con mediastinitis e infección de la herida quirúrgica. Dos años después acudió a su hospital de referencia por dolor en el hombro izquierdo de características osteomusculares, se administró analgesia convencional por vía venosa periférica y a las dos horas presentó pérdida brusca de conciencia e hipotensión. En el ECG practicado se observó supradesnivel del segmento ST en cara inferior (Figura 1a). Se realizó TC cerebral apreciándose embolia gaseosa cerebral a nivel de múltiples estructuras (Figura 1b). En el TC torácico se descartó la existencia de aire en estructuras mediastínicas. En la analítica destacaba leucocitosis de 22,600/mm3 con 90\% de neutrófilos, CK de $3606 \mathrm{U} / \mathrm{L}, \mathrm{CK}-\mathrm{MB}$ de $368 \mathrm{U} / \mathrm{L}$ y troponina I de $12 \mathrm{ng} / \mathrm{ml}$. Se realizó ecocardiograma objetivándose acinesia inferobasal sin apreciar foramen oval ni comunicación interauricular, siendo negativo el test salino.

En el TC cerebral de 6 días después se apreciaba infarto isquémico agudo en la totalidad del hemisferio cerebral derecho con edema y colapso del cuarto ventrículo, infartos isquémicos parietales y occipitales bilaterales en diferentes estadios evolutivos. La evolución posterior fue desfavorable produciéndose el exitus 48 horas después. La necropsia realizada confirmó la mediastinitis e isquemia cerebral y miocárdica, con arterias coronarias sin trombos ni lesiones significativas. Se estableció el diagnóstico de embolismo gaseoso arterial cerebral e infarto agudo de miocardio secundario a embolismo gaseoso en arterias coronarias.

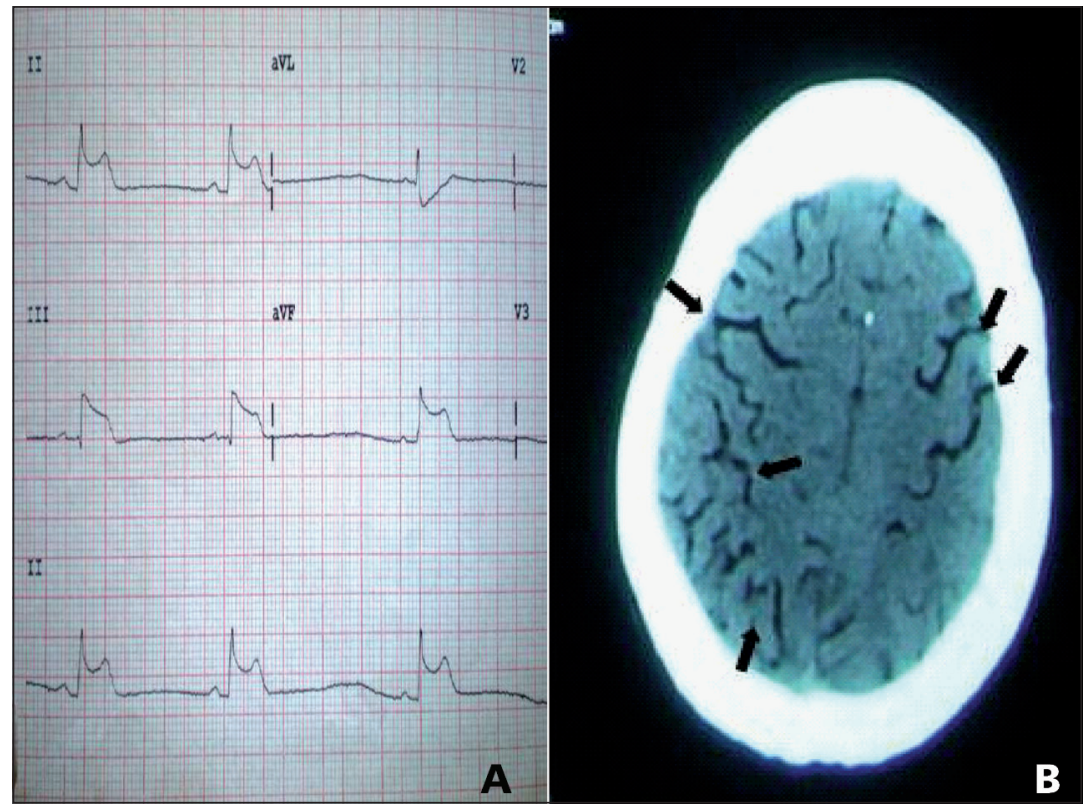

Figura 1. A: Supradesnivel del segmento ST en derivaciones: II, III y aVF. B: Presencia de aire a nivel de múltiples estructuras vasculares cerebrales. 
Las primeras referencias de los embolismos gaseosos vasculares fueron recogidas en el tratado de Morgagni, publicado en $1769^{1}$. Existen dos tipos de embolismos gaseosos: venosos y arteriales, diferenciados por el mecanismo de entrada y órganos afectados. La causa más común de embolismo venoso es a través de catéteres venosos centrales o periféricos, el gas es transportado hasta los pulmones a través de las arterias pulmonares.

Las consecuencias pueden ser alteraciones en el intercambio gaseoso, arritmias, hipertensión pulmonar, sobrecarga del ventrículo derecho o fallo cardíaco ${ }^{2}$. Los embolismos arteriales se producen por la entrada de gas en las venas pulmonares o directamente en las arterias de la circulación sistémica. El embolismo paradójico ocurre cuando la embolia procede de la circulación venosa y pasa a la arterial a través de un foramen oval permeable o malformaciones arteriovenosas pulmonares. Existen anestésicos que disminuyen la capacidad pulmonar de filtrar el gas de la circulación venosa ${ }^{3}$. Cuando la embolia gaseosa afecta a las arterias cerebrales se produce la muerte neuronal por isquemia y aparece una respuesta inflamatoria por irritación endotelial. Uno y otro derivarán en un edema cerebral vasogénico ${ }^{2}$. La afectación coronaria puede cursar con episodios de isquemia, arritmias, insuficiencia cardíaca o infarto masivo. El tratamiento se basa en mantener las funciones vitales y actuar sobre las complicaciones. La terapia con oxígeno hiperbárico mediante el incremento de la presión ambiental y la hiperoxia disminuye el tamaño de las burbujas y ha mostrado su efecto beneficioso, disminuyendo el edema cerebral ${ }^{4}$.

José Luis Cabrerizo-García ${ }^{1,}$ Begoña Zalba-Etayo ${ }^{2}$, Luis Martín-Villen ${ }^{2}$. ${ }^{1}$ Servicio de Medicina Interna. ${ }^{2}$ Servicio de Medicina Intensiva. Hospital Clinico Universitario Lozano Blesa. Zaragoza, España.

\section{Referencias}

1. Fries CC, Levowitz B, Adler S, Cook AW, Karlson KE, Dennis C. Experimental cerebral gas embolism. Ann Surg 1957; 145: 61-470.

2. Muth CM, Shank ES. Gas embolism. N Engl J Med 2000; 342: 476-482.

3. Katz J, Leiman BC, Butler BD. Effects of inhalation anaesthetics on filtration of venous gas emboli by the pulmonary vasculature. Br J Anaesth 1988; 61: 200-5.

4. Blanc P, Boussuges A, Henriette K, Sainty JM, Deleflie M. Iatrogenic cerebral air embolism: importance of an early hyperbaric oxygenation. Intensive Care Med. 2002; 28: 559-63.

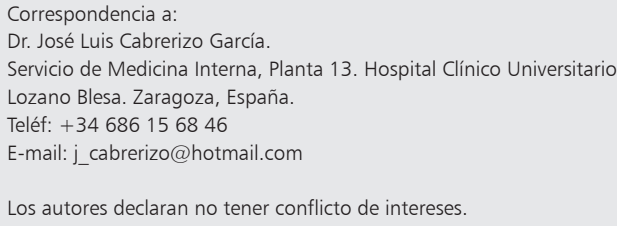

